\title{
A CONSTITUTIVE FRAMEWORK FOR HUMAN DERMIS MECHANICAL MODELLING
}

\author{
ALESSANDRA ALDIERI ${ }^{1}$, MARA TERZINI ${ }^{1}$, CRISTINA BIGNARDI $^{1}$, ELISABETTA M. $_{\text {ZANETTI }}^{2}$ \\ \& ALBERTO L. AUDENINO ${ }^{1}$ \\ ${ }^{1}$ DIMEAS, Politecnico di Torino, Italy \\ ${ }^{2}$ Department of Engineering, University of Perugia, Italy
}

\begin{abstract}
Finite element models, in conjunction with adequate constitutive relations of the materials involved, have proved to be crucial in many medical applications, such as in surgical planning. Nevertheless, a thorough numerical analysis of dermis' mechanical response is a challenging research area because of dermis' highly anisotropic and nonlinear behaviour. The aim of this work has been to assess the performance of two orthotropic and one isotropic hyper-elastic constitutive laws, providing an experimental-computational framework for the definition of reliable constitutive models of dermis tissue. Experimental, equi-biaxial stress-strain data obtained on human reticular dermis specimens have been exploited in order to extract, through a stochastic optimization procedure, constitutive parameters of three widely used constitutive laws: the Ogden, Holzapfel and Gasser-Ogden-Holzapfel (GOH) models. A set of specimen specific parameters and a set of best matching parameters, determined by simultaneously fitting all experimental stress-strain curves, have been obtained. The goodness of the selected laws has been assessed by means of FEM simulations performed in Abaqus (Simulia, Dessault Systèmes Inc.) which reproduce the actual specimen boundary conditions and geometry, rebuilt through an image segmentation process implemented in MATLAB (The MathWorks, Inc.). Models have been validated comparing experimental and numerical outcomes related to reference points on the specimen surface. In the preliminary fitting phase, Normalized Root Mean Square Error values were above 0.9 for the specimen-specific and above 0.54 for the best matching models. The comparison between numerical and experimental outcomes has highlighted the inadequacy of the isotropic constitutive law in reproducing the dermis behaviour, particularly at higher stretch levels. Errors obtained in the horizontal loading direction are lower than $40 \%$ for Holzapfel and GOH models, while the Ogden model reaches the $80 \%$. Lower and more uniform errors occur in the orthogonal direction, which settles below $30 \%$ for the orthotropic laws, while it increases up to $40 \%$ in the isotropic case.
\end{abstract}

Keywords: human dermis, anisotropy, biaxial data, FE analysis, constitutive model validation.

\section{INTRODUCTION}

Skin represents an extremely complex organ, organized in mainly two-dimensional superimposed structures. With its total surface occupying about $2 \mathrm{~m}^{2}$, it is one of the largest organ of the human body. To fulfil its many functions, it undergoes a wide range of mechanical, chemical, physical and biological stresses. The skin is composed of three layers: the epidermis $(0.1 \mathrm{~mm}-1.5 \mathrm{~mm})$, an intermediate thicker layer known as dermis $(1 \mathrm{~mm}-4 \mathrm{~mm})$, and the hypodermis. It consists mainly of collagen (approximately $75 \%$ of the dry weight) and elastin (4\% of the dry weight) fibers embedded in a highly-hydrated proteoglycan ground matrix [1]. Dermis, being the thickest layer, represents the skin main component. Although it can be further divided in papillary, adjacent to epidermis, and reticular dermis, it is the reticular dermis which occupies the majority of the total thickness. This reticular layer provides the most significant contribution to the overall mechanical properties of skin [2]. Skin, as well as other human soft tissues [3], shows a nonlinear mechanical response, which is mainly attributable to its microstructure. In fact, when stretched, the collagen fibers, initially crimped at low strains, are progressively oriented until they get completely aligned with the direction of the load. Hence, the stress-strain response 
is characterized by a low modulus initial phase, followed by a significant stiffening caused by the fibers gradual reorientation. The collagen fibers network is responsible also for the anisotropic behavior of dermis, since a dominant fiber orientation is present, which affects the mechanical response of skin [4]. Skin mechanical properties have been investigated in vitro mainly by means of uniaxial and biaxial tests as well as through bulge tests methods [5]. Though authors have highlighted the challenges unique to biological tissues related to the planning and the performance of biaxial experiments, the limitations concerning the inadequacy of uniaxial tests in fully reproducing the in situ loading of soft tissues and in disclosing their profound anisotropy has been enhanced [6], [7]. Hence, both uniaxial [2], [4], [8]-[11] and biaxial [12]-[16] tensile tests are still being adopted aiming at investigating the mechanical properties of collagenous tissues, often in conjunction [6], [17]. The characterization of tissues mechanical response turns out to be fundamental also for the development of constitutive models and the subsequent definition of material properties exploitable in Finite Element (FE) studies. FE models in conjunction with adequate constitutive relations would indeed be pivotal in several physiological and medical applications related to both native and engineered tissues [18], allowing to predict their mechanical response under untested loading states: this is the reason why constitutive modelling of soft biological tissues still represents an active research area [14]. However, proper constitutive relations and their respective parameters need to be selected in order to get reliable results from numerical simulations. Numerical methods applied to soft biological tissues have considerably spread recently, and, as a consequence, hyper-elastic anisotropic constitutive formulations have been implemented in commercial FE codes. Although nonlinear isotropic material models such as the Ogden, Mooney-Rivlin and Neo-Hookean ones have been used to describe the mechanical response of skin [9], [10], [19], more adequate anisotropic structural formulations, i.e. accounting for the real collagenous structure of soft tissues, have recently been developed [7], [15], [16], [20], [21], which more coherently represent their mechanical properties.

The main objective of this work has been the implementation of two structural anisotropic material models available in literature and one isotropic formulation for modelling the mechanical behavior of human reticular dermis. Biaxial stress-strain data from four specimens have been exploited to extract each model parameters through a stochastic optimization procedure. Afterwards, the implemented models have been validated comparing FE analyses results to experimental displacements distributions. The last step was considered mandatory to set up reliable models, able to faithfully reproduce the actual tissue mechanical response that is crucial for the design and the evaluation of medical and surgical procedures.

\section{MATERIALS AND METHODS}

\subsection{Experimental biaxial data}

Biaxial experimental data for model parameters extraction and for biaxial testing simulations are described in detail in the work by Terzini [23], and will be here briefly explained.

Large strips of dermis tissue from the lower back of a human donor were dissected along the anatomical cranio-caudal (CC) direction, and four specimens per donor were obtained by cutting $30 \mathrm{~mm} \times 30 \mathrm{~mm}$ squares from the strips, coherently with the anatomical orientations identified in the original tissue. The specimens considered in this work had not undergone any decellularization treatment, but had been preserved at $85 \%$ glycerol; prior to testing, they were all washed in order to remove all glycerol. 
Equi-biaxial tensile tests were performed through a custom designed fixture, integrated in the available uniaxial testing machine (Bose Electroforce ${ }^{\circledR} 3200$ ), in which the specimen was gripped by three hooks per side. The two orthogonal loading directions corresponded to the anatomical cranio-caudal (CC) and medio-lateral (ML) directions, which will be also referred to as horizontal and vertical respectively, according to experimental tests. The loading rate was set to $0.16 \mathrm{~mm} / \mathrm{s}$ and the test was stopped when a $10 \mathrm{~mm}$ displacement was achieved on both axes. Images of the specimen undergoing the test were captured with a $1 \mathrm{~Hz}$ frame-rate by means of a full-frame digital camera (Canon EOS 5D Mark II) equipped with an autofocus lens for macro photography (Canon EF 100mm f/2.8 Macro USM). Forces along the two orthogonal tensile directions were measured by two load cells; Cauchy stresses were calculated dividing load cell readings by the relative cross-sectional area of the specimen.

Engineering strains were determined by tracking the average distance along the $\mathrm{CC}$ and ML directions of four central markers drawn on the specimen's surface (Fig. 1). Experimental stress-strain curves of the four specimens are presented in Fig. 2.

The experimental stress-strain curves (Fig. 2) resulted to have a high variability, and demonstrated the pronounced anisotropy of the tissue, since CC and the ML directions have produced very different responses; as a consequence, anisotropic hyper-elastic material models were expected to be more adequate to reproduce dermis response. Two anisotropic hyper-elastic constitutive laws were selected, which had been developed for modelling arterial walls but can be adapted to any soft tissue, together with an isotropic hyper-elastic model which still finds application in soft tissues modelling [10], [19]. The isotropic Ogden model strain energy function presents as follows:

$$
W=\sum_{i=1}^{N}\left(\overline{\lambda_{1}^{\alpha_{l}}}+\overline{\lambda_{2}^{\alpha_{l}}}+\overline{\lambda_{3}^{\alpha_{\imath}}}-3\right) \frac{2 \mu_{i}}{\alpha_{i}{ }^{2}}+\sum_{i=1}^{N} \frac{1}{D_{i}}(J-1)^{2 i},
$$

where $J=\operatorname{det}(F)$, with $F$ being the deformation gradient. $\lambda_{1}, \lambda_{2}, \lambda_{3}$ are the principal stretches, for which, if incompressibility is assumed, $\lambda_{1} \lambda_{2} \lambda_{3}=1$ holds. $N$ is related to the model order. Coefficients $\mu_{i}, \alpha_{i}$ are material parameters to be determined through the fitting procedure.

The anisotropic Holzapfel [7], Holzapfel et al. [21] and Gasser-Ogden-Holzapfel (GOH) [20] laws are structural invariant-based models both assuming the presence of two families of collagen fibers affecting the mechanical response. Holzapfel law strain energy function reads as follows:

$$
W=\frac{c}{2}\left(\overline{I_{1}}-3\right)+\frac{k_{1}}{2 k_{2}} \sum_{i=4,6}\left\{\exp \left[k_{2}\left(\overline{I_{l}}-1\right)^{2}\right]-1\right\},
$$

where $c, k_{1}>0$ are stress-like parameters, $k_{2}$ is a dimensionless parameter. $I_{1}$ is the first
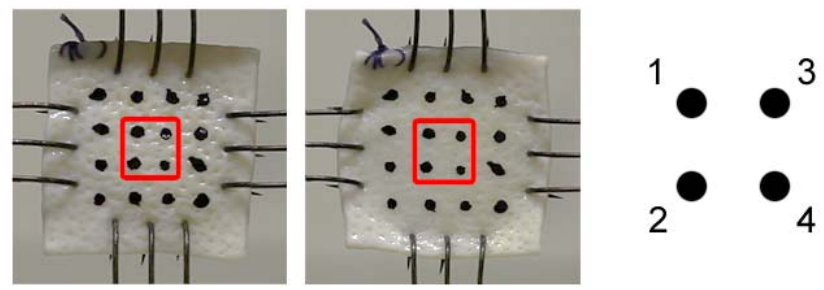

Figure 1: An image of the specimen in the unloaded and loaded configurations. Numbers assigned to central reference points are highlighted. 

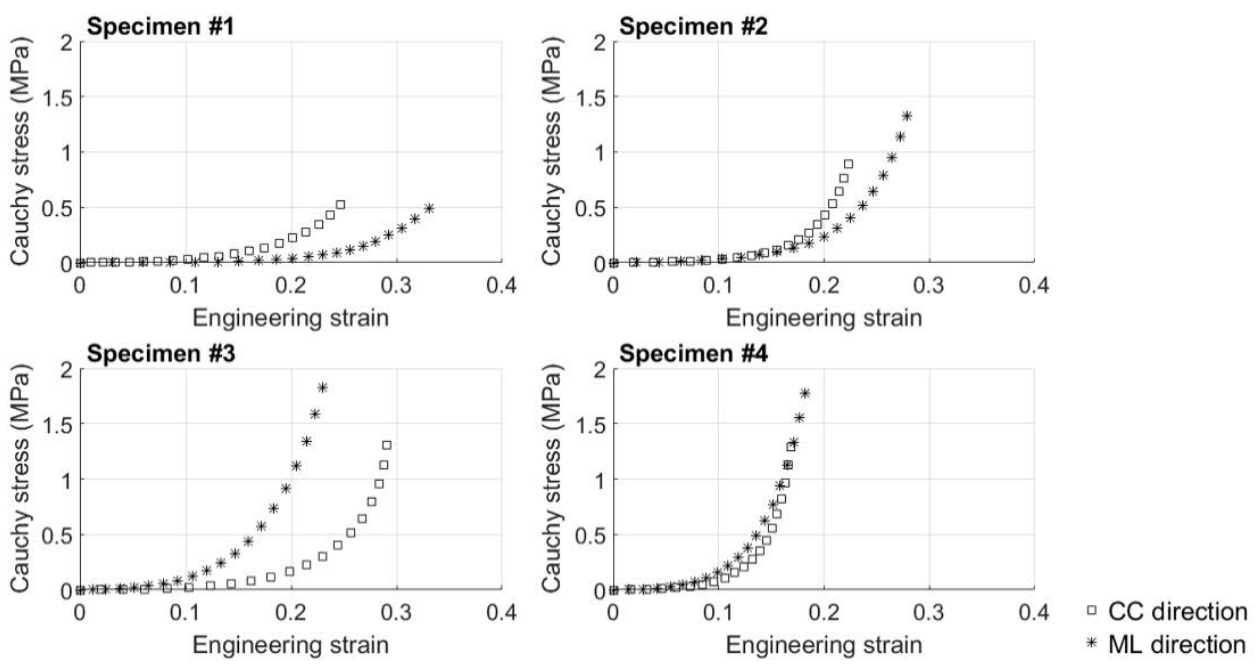

Figure 2: Human dermis specimens stress-strain experimental curves for the four specimens.

invariant of the modified Cauchy-Green tensor $\bar{C}$ and is associated to the isotropic contribution given by the extracellular matrix; invariants $\overline{I_{4}}$ and $\overline{I_{6}}$ on the other hand, which have a definite physical meaning, relate to the anisotropic contribution, and are the squares of the stretches in the fibres directions. They are defined as $\overline{I_{4,6}}=\overline{\boldsymbol{C}}: \boldsymbol{a}_{01,2} \otimes \boldsymbol{a}_{01,2}, \overline{\boldsymbol{C}}$ being the modified elastic right Cauchy-Green tensor. Note that in the hypothesis of incompressibility $\overline{\boldsymbol{C}}=\boldsymbol{C}$. Whereas Holzapfel constitutive law assumes collagen fibers to be perfectly aligned, $\mathrm{GOH}$ model introduces an additional parameter able to model fibers dispersion along a preferred direction and therefore reproduces more realistically the actual dermis histology. The GOH strain energy function reads as:

$$
W=\frac{c}{2}\left(\overline{I_{1}}-3\right)+\frac{k_{1}}{2 k_{2}} \sum_{i=4,6}\left[\exp \left\{k_{2}\left[\kappa \overline{I_{1}}+(1-3 \kappa) \overline{I_{l}}-1\right]^{2}\right\}-1\right]
$$

The dispersion parameter $\kappa$, assumed to characterize the two fibers families in the same way, ranges from 0 to $1 / 3$, with $\kappa=0$ describing a perfect alignment, and $\kappa=1 / 3$ representing an isotropic distribution of fibers along their preferential orientation.

\subsection{Identification of constitutive parameters}

The extraction of the constitutive parameters for each analyzed model was based on the comparison between the model-predicted and experimental stress-strain values. The objective function, accounting for the difference between the predicted and experimental stress values in each direction, was progressively minimized, in order to compute the parameters providing the best match. The objective function was expressed as:

$$
\Pi(\boldsymbol{\alpha})=\sqrt{\frac{1}{N} \sum_{i=1}^{N}\left|\sigma_{C C i}^{\exp 2}-\sigma\left(\boldsymbol{\alpha}, \varepsilon_{C C i}^{\text {exp }}\right)_{C C i}^{\bmod 2}\right|+\left|\sigma_{M L i}^{\exp 2}-\sigma\left(\boldsymbol{\alpha}, \varepsilon_{C C i}^{\exp }\right)_{M L i}^{\bmod 2}\right|},
$$

where $\alpha$ represents the set of unknown constitutive parameters, $N$ the number of experimental data, $\sigma^{\exp }$ and $\sigma^{\text {mod }}$ the experimental and the model predicted stress values respectively. 
Since experimental data were available along the two orthogonal loading directions (CC and ML directions), the anisotropic constitutive laws were defined as orthotropic models. Being this cost function (eqn (1)) characterized by a complex behavior, a stochastic algorithm instead of a deterministic one was selected for the optimization problem solution, in order to avoid falling into a local minimum [22]. Hence, the simulated annealing algorithm was adopted to solve a minimization problem, and the simulannealbnd MATLAB function was used. The first optimization procedure has been performed considering the stress-strain curve of one specimen, extracting a specimen-specific parameter set; subsequently, the whole set of experimental curves has been used simultaneously to determine a new set of parameters able to guarantee the best-fit for a generic material model.

\subsection{Finite Element simulations and validation}

Material models have been implemented in the FE software Abaqus Standard 6.13 using the previously extracted constitutive parameters.

An accurate reproduction of the actual specimen and of its boundary conditions was pivotal: a segmentation process was therefore performed on the images captured during testing. The image of the unloaded specimen allowed both the identification of the hooks insertion points, so that boundary conditions could be applied on the corresponding nodes of the geometric model, and the detection of the initial position of the central markers, in order to identify their equivalent nodes. Furthermore, the segmentation of the following images provided the progressive experimental displacements of central markers in the CC and ML directions, which had to be compared to the corresponding numerical outcomes. Length, height and width have been assigned to the geometric model coherently with the original specimen size; boundary conditions were given as prescribed displacements of the previously selected nodes, with a displacement rate of $0.16 \mathrm{~mm} / \mathrm{s}$. Eight-node biquadratic plane elements (Abaqus element type CPS8) with size of $0.3 \mathrm{~mm}$ have been used for all simulations. Static simulations accounting for nonlinearity due to both the constitutive model and large deformations have been performed for a time lag of $45 \mathrm{~s}$ and keeping the default Abaqus convergence criteria unaltered.

Validation of each model was carried out comparing the experimental displacement of the central markers along CC and ML directions to those experienced by the corresponding nodes during simulations. Relative errors were computed for each reference point along CC and ML directions according the formulation:

$$
e_{r e l}(i)=\frac{d^{\text {num }}(i)-d^{e x p}(i)}{d^{\exp }(\text { end })}
$$

where $d^{\text {num }}(i)$ and $d^{\text {exp }}(i)$ represent the numerical and experimental coordinates respectively at the $i$-th time instant, and $d^{\exp }$ (end) refers to the final experimental position of the markers. Relative errors arrays were obtained for each reference point and related to the two orthogonal loading directions.

\section{RESULTS AND DISCUSSION}

This study aimed to numerically assess the performance of three constitutive models in reproducing the mechanical behavior of human reticular dermis. Therefore, once constitutive parameters had been extracted fitting stress-strain curves, numerical simulations replicating the equi-biaxial tests have been performed and the respective outcomes have been compared to experimental findings. 
With reference to the preliminary fitting phase, Table 1 reports Ogden constitutive parameters sets both for the specimen-specific model and the generic material model. A firstorder model has been chosen since it proved to be sufficient to adequately fit experimental data, and therefore there was no reason to implement more complex models. As a consequence, only two parameters needed to be determined. NRMSE between predicted and experimental data are reported as well in Table 1.

Table 2 and Table 3 show Holzapfel and GOH constitutive model coefficients, together with respective NRMSE values. It should be emphasized that parameters $\gamma$, defining symmetric fiber orientations with respect to the horizontal axis (in this case the CC direction), and $\kappa$, have been deduced from the macroscopic mechanical response rather than from the underlying histology of the dermis. To the authors' knowledge, the only works in which one of the selected constitutive models had been similarly adopted for dermis structural modelling are those by Tonge et al. [5] and Ní Annaidh et al. [8], where GOH model was adopted. Discrepancies arise between the here identified material parameters and those proposed by Tonge et al. as far as $k_{1}$ and the ratio $\frac{k_{1}}{k_{2}}$ are concerned, whereas better agreement is found with respect to Ní Annaidh et al. This could be explained accounting for the marked differences between experimental data used as input for parameters extraction. Tonge et al. performed bulge tests, and analytically determined stress state modelling the deformed specimen as a thin shell. On the contrary, in this work, similarly to Ní Annaidh et al., tensile tests were performed, therefore the stress state was directly related to the definition of a crosssectional area.

Moreover, both Holzapfel and GOH computed material parameters have the same orders of magnitude as those presented by Gasser at al. [20] and Holzapfel et al. [21], although they were obtained for arterial wall constitutive material.

Fig. 3 displays the stress-strain curves predicted by the three models compared to experimental ones. Ogden model, being an isotropic constitutive law, is characterized by a unique stress-strain curve. As visible, with reference to specimen-specific experimental data, all constitutive models provide an excellent fit of the stress-strain curves. Nonetheless, when the whole set of stress-strain data is considered to compute generic material coefficients, the accurate prediction of the experimental output becomes more demanding.

Table 1: Ogden constitutive parameters.

\begin{tabular}{|c|c|c|c|}
\hline Specimens & $\mu(\mathrm{MPa})$ & $\alpha$ & NRMSE \\
\hline Specimen 1 & $7.13 \mathrm{e}^{-3}$ & -10.86 & 0.99 \\
\hline Generic material model & $1.61 \mathrm{e}^{-2}$ & -13.26 & 0.64 \\
\hline
\end{tabular}

Table 2: Holzapfel constitutive parameters.

\begin{tabular}{|c|c|c|c|c|c|}
\hline Specimens & $k_{1}(\mathrm{MPa})$ & $k_{2}$ & $c(\mathrm{MPa})$ & $\gamma\left(^{\circ}\right)$ & NRMSE \\
\hline Specimen 1 & 0.02 & 1.77 & 0.01 & 39.14 & 0.90 \\
\hline Generic Material model & 0.02 & 2.37 & 0.01 & 33.10 & 0.61 \\
\hline
\end{tabular}


Table 3: Gasser-Ogden-Holzapfel constitutive parameters [20].

\begin{tabular}{|c|c|c|c|c|c|c|}
\hline Specimens & $k_{1}(\mathrm{MPa})$ & $k_{2}$ & $c(\mathrm{MPa})$ & $\kappa$ & $\gamma\left({ }^{\circ}\right)$ & NRMSE \\
\hline Specimen 1 & 0.51 & 3.99 & 0.01 & 0.29 & 41.86 & 0.98 \\
\hline $\begin{array}{c}\text { Generic } \\
\text { Material model }\end{array}$ & 2.2 & 0.86 & 0.01 & 0.27 & 45.5 & 0.54 \\
\hline
\end{tabular}
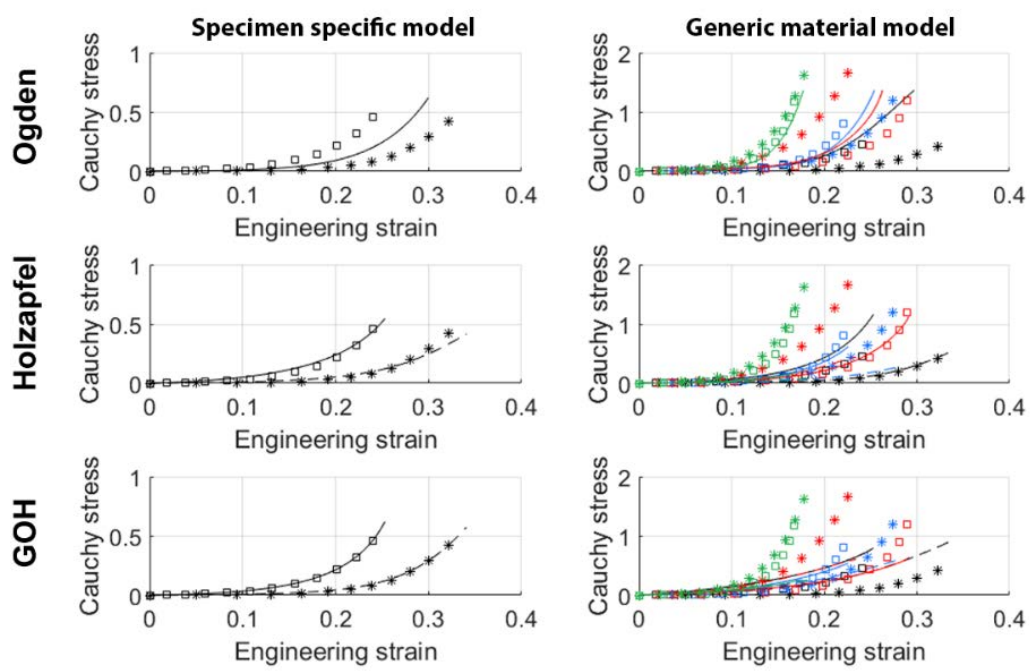

- specimen \#1

- specimen \#2

specimen \#3

- specimen \#4

Figure 3: Experimental and model predicted Cauchy stress (MPa) - Engineering strain data for the specimen specific parameters (left) and for the generic material model ones (right); ' $\square$ ': experimental CC data; '*': experimental ML data; solid line: model predicted CC data; dashed line: model predicted ML data.

As previously explained, the constitutive models were validated by means of FE analyses, which allowed to compare the displacements of four central markers with those experienced by corresponding nodes belonging to the numerical model. Fig. 4 and Fig. 5 display relative errors at each reference point along the CC and ML directions respectively as a function of the stretch ratio $\lambda$ at the hooks insertion points.

Errors are generally higher along the CC direction than along the ML one, and one marker along the $\mathrm{CC}$ direction (no 3 ) is characterized by significantly higher errors. Focusing on the displacements experienced by the four markers in the CC direction (Fig. 6), tracked through test images segmentation, it is possible to observe how marker 3 actually displaces less than marker 1 , while it should move symmetrically. It also moves less than marker 4 , which is closer to the lower fixed hooks. 
Experimental displacements of markers were actually identified tracking only their centroids. However, not only was markers size considerably bigger than a single point, but markers also underwent deformation during testing. As a consequence, whenever deformation was significantly greater than displacement, centroids coordinates might not change substantially. For example, a null displacement of marker 3 centroid was detected between the $10^{\text {th }}$ and $30^{\text {th }}$ second. The inability of the numerical displacements to reproduce the experimental displacements could then be due to approximations made during the segmentation process rather than to an inaccurate material model.

Hence, if outcomes related to marker 3 are neglected and the goodness of the constitutive models are compared, Ogden isotropic model turns out to be characterized by the highest errors, especially in the CC direction. Indeed, along the CC direction it reaches the $80 \%$ while the orthotropic models settle below the $40 \%$, with slight differences between them. In the ML direction errors produced by all the models are characterized by a much more uniform pattern, where the Ogden model reaches $40 \%$ errors and the orthotropic one settles below $30 \%$.

As for the subtle differences between the structural models, if the Holzapfel constitutive law reproduces better the experimental outcomes in the $\mathrm{CC}$ direction, it is the $\mathrm{GOH}$ one which generally produces lower errors along the orthogonal loading direction.

The general performances of all models have been evaluated removing 'marker 3' outlier and averaging errors values (Fig. 7). The Ogden model has resulted to give the poorest performance with reference to human dermis subjected to equi-biaxial solicitation. Conversely, Holzapfel and GOH models settle, on average, below 30\% along both the loading directions, whereas Ogden model reaches $60 \%$ (CC direction). The most significant differences are found in the $\mathrm{CC}$ direction, where Holzapfel model has produced the lowest errors $(-10 \%$ with reference to $\mathrm{GOH}$ model).

(a)
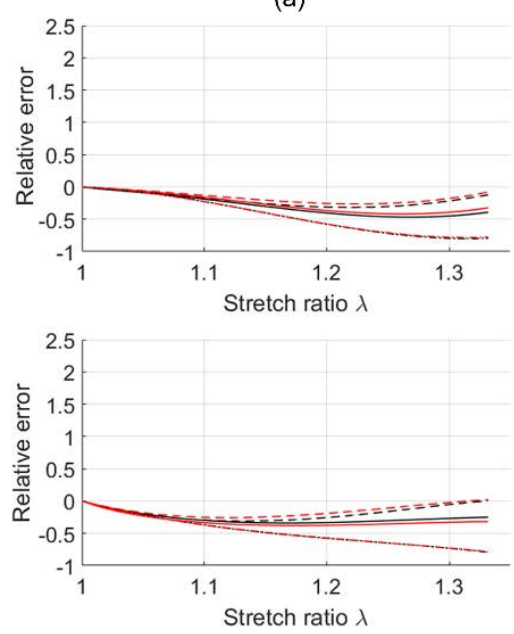

(b) (c)
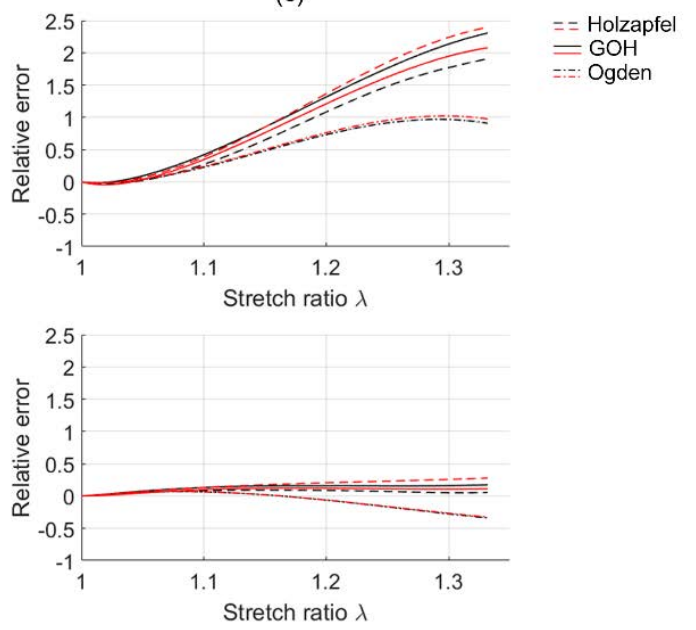

(d)

Figure 4: Comparison of relative errors at the four reference markers in the CC direction; (a): marker 1, (b): marker 2, (c): marker 3, (d): marker 4. In black, specimenspecific fitted models are depicted, in red, the best-fitting ones. 
(a)
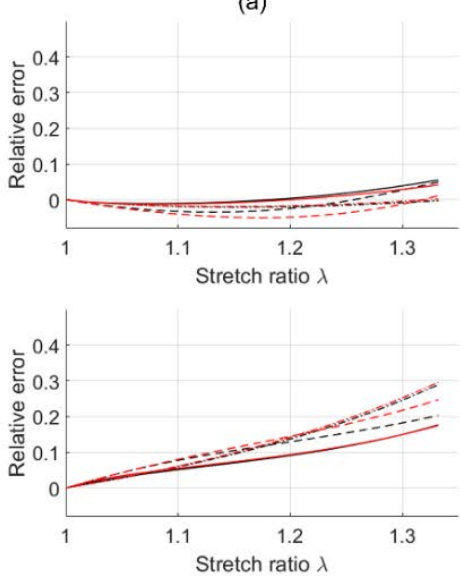

(b) (c)

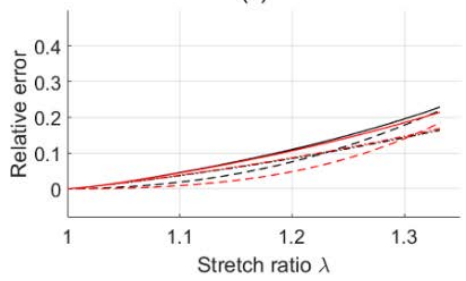

$==$ Holzapfe

$=\mathrm{GOH}$

=:: Ogden

(d)

Figure 5: Comparison of relative errors at the four reference markers in the ML direction; (a): marker 1, (b): marker 2, (c): marker 3, (d): marker 4. In black, specimenspecific fitted models are depicted, in red, the best-fitting ones.

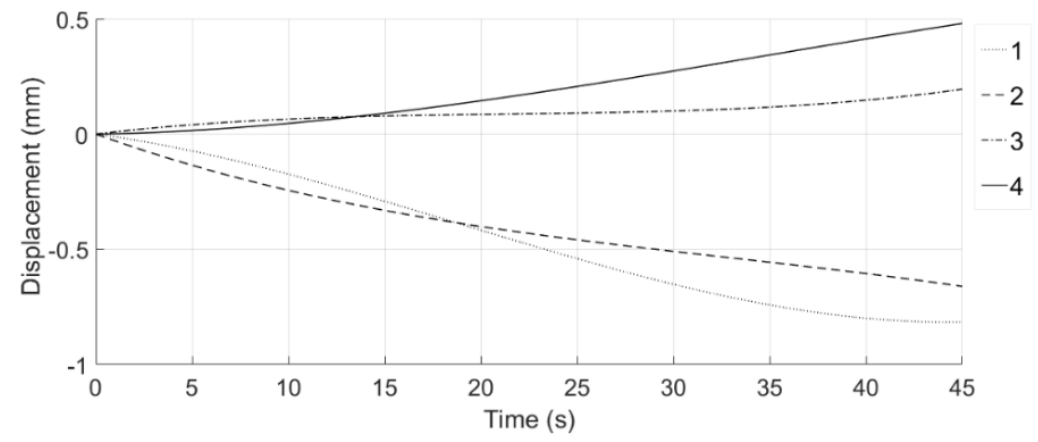

Figure 6: Experimental marker displacements from tests image segmentation.

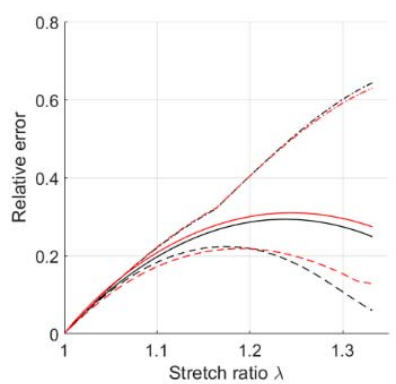

(a)

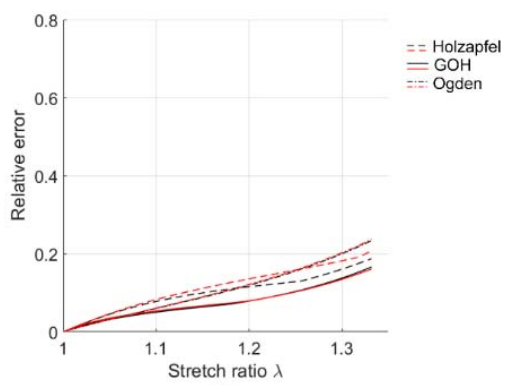

(b)

Figure 7: Averaged error absolute values along CC direction (a) and ML direction (b). In black specimen-specific fitted models are depicted, in blue the best-fitting ones. 


\section{CONCLUSION}

The object of this paper has been identifying a constitutive model able to describe the mechanical behavior of human reticular dermis. Three constitutive laws have been selected from literature concerning soft tissues constitutive modelling: one isotropic and two structural anisotropic formulations. Experimental equi-biaxial stress-strain data related to four specimens excised from the lower back of the same donor have been used to fit the models, extract material parameters and as a benchmark to test the performance of finite element models in forecasting full-field displacement. More in detail, FE analyses have then been implemented to replicate equi-biaxial tests and to compare numerical and experimental displacements experienced by four central markers along the two loading directions. Discrepancies between experimental and numerical outcomes have pointed out the inadequacy of the isotropic Ogden model. Both anisotropic formulations produced a better matching between numerical and experimental outcomes, being characterized by relative errors which settle, on average, below 30\%. These discrepancies can be ascribed to assumptions taken in the modelling procedure and limitations of the experimental set up. In fact, the dermis model has been simplified, having assumed it is an incompressible and homogeneous material. Besides, the segmentation process might have led to inaccuracies, since marker displacements were tracked through their centroids, but they deformed during testing.

This work adopts and proposes an experimental-computational procedure aimed to develop reliable constitutive models, to be used for medical and surgical evaluations. The identification of the material models has been performed also considering more than one specimen in order to take into account the variability of material parameters even in specimens coming from the same area of the same donor. Secondly, the validation procedure has been performed considering full-field displacement and not the only full-specimen stressstrain curve, as reported more frequently in literature.

\section{REFERENCES}

[1] Li, W., Modelling methods for In vitro biomechanical properties of the skin: A review. Biomedical Engineering Letters, 5(4), pp. 241-250, 2015.

[2] Terzini, M., Bignardi, C., Castagnoli, C., Cambieri, I., Zanetti, E.M. \& Audenino, A.L., Dermis mechanical behaviour after different cell removal treatments. Medical Engineering \& Physics, 38(9), pp. 862-869, 2016.

[3] Zanetti, E.M., Perrini, M., Bignardi, C. \& Audenino, A.L., Bladder tissue passive response to monotonic and cyclic loading. Journal of Biorheology, 49(1), pp. 49-63, 2012.

[4] Ní Annaidh, A. et al., Characterization of the anisotropic mechanical properties of excised human skin. Journal of the mechanical behaviour of biomedical materials, 5(1), pp. 139-148, 2012.

[5] Tonge, T.K., Voo, L.M. \& Nguyen, T.D., Full-field bulge test for planar anisotropic tissues: Part II-a thin shell method for determining material parameters and comparison of two distributed fiber modelling approaches. Acta Biomaterialia, 9(4), pp. 59265942, 2013.

[6] Cooney, G.M., Moerman, K.M., Takaza, M., Winter, D.C. \& Simms, C.K., Uniaxial and biaxial mechanical properties of porcine linea alba. Journal of the Mechanical Behaviour of Biomedical Materials, 41, pp. 62-82, 2015.

[7] Holzapfel, G.A., Biomechanics of soft tissue. The Handbook of Materials Behaviour Models, 3, pp. 1049-1063, 2001. 
[8] Ní Annaidh A. et al., Automated estimation of collagen fibres dispersion in the dermis and its contribution to the anisotropic behaviour of skin. Annals of Biomedical Engineering, 40(8), pp. 1666-1678, 2012.

[9] Groves, R.B., Coulman, S.A., Birchall, J.C. \& Evans, S.L., An anisotropic, hyperelastic model for skin: experimental measurements, finite element modelling and identification of parameters for human and murine skin. Journal of Mechanical Behaviour of Biomedical Materials, 18, pp. 167-180, 2013.

[10] Karimi, A., Navidbakhsh, M., Haghighatnama, M. \& Haghi, A.M., Determination of the axial and circumferential mechanical properties of the skin tissue using experimental testing and constitutive modelling. Computer Methods in Biomechanics and Biomedical Engineering, 18(16), pp. 1768-1774, 2015.

[11] Ottenio, M., Tran, D., Annaidh, A.N., Gilchrist, M.D. \& Bruyère, K., Strain rate and anisotropy effects on the tensile failure characteristics of human skin. Journal of the Mechanical Behaviour of Biomedical Materials, 41, pp. 241-250, 2015.

[12] Bellini, C., Glass, P., Sitti, M. \& Di Martino, E.S., Biaxial mechanical modelling of the small intestine. Journal of the Mechanical Behaviour of Biomedical Materials, 41, pp. 68-82, 2011.

[13] Natali, A.N., Audenino, A.L., Artibani, W., Fontanella, C.G., Carniel, E.L. \& Zanetti, E.M., Bladder tissue biomechanical behavior: Experimental tests and constitutive formulation. Journal of Biomechanics, 48, pp. 3088-3096, 2015.

[14] Fan, R. \& Sacks, S., Simulation of planar soft tissues using a structural constitutive model finite element implementation and validation. Journal of Biomechanics, 47(9), pp. 2043-2054, 2014.

[15] Sommer, G. et al., Multiaxial mechanical properties and constitutive modelling of human adipose tissue: a basis for preoperative simulations in plastic and reconstructive surgery. Acta Biomaterialia, 9(11), pp. 9036-9048, 2013.

[16] Sommer, G. et al., Multiaxial mechanical response and constitutive modelling of esophageal tissues: impact on esophageal tissue engineering. Acta Biomaterialia, 9(11), pp. 9379-9391, 2005.

[17] Lyons, M., Winter, D.C. \& Simms, C.K., Mechanical characterisation of porcine rectus sheath under uniaxial and biaxial tension. Journal of Biomechanics, 47(8), pp. 18761884, 2014.

[18] Sacks, M.S., Biaxial mechanical evaluation of planar biological materials. Journal of Elasticity and the Physical Science of Solids, 61(1-3), pp. 199-246, 2000.

[19] Flynn, C., Stavness, I., Lloyd, J. \& Fels, S., A finite element model of the face including an orthotropic skin model under in vivo tension. Computer Methods in Biomechanics and Biomedical Engineering, 18(6), pp. 571-582, 2015.

[20] Gasser, T.C., Ogden, R.W. \& Holzapfel, G.A., Hyper-elastic modelling of arterial layers with distributed collagen fibre orientation. Journal of the Royal Society Interface, 3(6), pp. 15-35, 2006.

[21] Holzapfel, G.A., Gasser, T.C. \& Ogden, R.W., A new constitutive framework for arterial wall mechanics and a comparative study of material models. Journal of Elasticity and the Physical Science of Solids, 61(1-3), pp. 1-48, 2000.

[22] Natali, A.N., Carniel, E.L., Pavan, P.G., Dario, P. \& Izzo, I., Hyper-elastic models for the analysis of soft tissue mechanics: definition of constitutive parameters. The First IEE/RASEMBS International Conference on Biomedical Robotics and Biomechatronics, pp. 188-191, 2006. 
378 Materials and Contact Characterisation VIII

[23] Terzini, M., Exploring the mechanical properties of ex vivo human dermis in vitro and in silico (Doctoral thesis, Politecnico di Torino, Turin, Italy). 2016, Online. http://porto.polito.it/id/eprint/2645217. Accessed on: 1 Mar. 2017. 